\title{
Reprogramming of endogenous Müller cells into photoreceptor-like cells induced by small-molecule compounds in mice
}

\section{Yuya Fujii}

Kyushu University Graduate School of Medical Sciences

Mitsuru Arima ( $\sim$ m-arima@eye.med.kyushu-u.ac.jp)

Kyushu University Graduate School of Medical Sciences

\section{Yusuke Murakami}

Kyushu University Graduate School of Medical Sciences

Koh-Hei Sonoda

Kyushu University Graduate School of Medical Sciences

\section{Article}

Keywords: Müller cells (MCs), RNA of rhodopsin (Rho), age-related macular degeneration (AMD), retinitis pigmentosa (RP)

Posted Date: September 17th, 2021

DOI: https://doi.org/10.21203/rs.3.rs-890527/v1

License: (c) (i) This work is licensed under a Creative Commons Attribution 4.0 International License. Read Full License 
Yuya Fujii, ${ }^{1}$ Mitsuru Arima, ${ }^{*} 1,2$ Yusuke Murakami, ${ }^{1}$ Koh-Hei Sonoda ${ }^{1}$

6

$7{ }^{1}$ Department of Ophthalmology, Graduate School of Medical Sciences, Kyushu University,

8 Fukuoka, Japan

$9 \quad{ }^{2}$ Center for Clinical and Translational Research, Kyushu University Hospital, Fukuoka, Japan

10 *Corresponding author: Mitsuru Arima

11 Department of Ophthalmology, Graduate School of Medical Sciences, Kyushu University, 3-

12 1-1 Maidashi, Higashi-ku, Fukuoka City, Fukuoka, Japan

13 Tel: +81-92-642-5648, Fax: +81-92-642-5663.

14 Email: m-arima@eye.med.kyushu-u.ac.jp 


\section{Abstract}

2 Lifelong visual impairment occurs from retinal diseases due to the inability of photoreceptor

3 cells to regenerate in mammals. We demonstrated that endogenous Müller cells (MCs) in

4 mice differentiate into photoreceptor-like cells by intravitreal injection of four small-molecule

5 compounds: tumor growth factor- $\beta$ inhibitor, bone morphogenetic protein inhibitor, glycogen

6 synthase kinase 3 inhibitor, and $y$-secretase inhibitor. In vitro, the messenger RNA of

7 rhodopsin (Rho) in MCs increased 30 -fold, and $25 \%$ of cultured MCs expressed Rho protein

87 days after stimulation with these compounds. In vivo, Rho-positive cells were regenerated

9 on day 7 after the intravitreal injection of four compounds, accompanied with recovery of

10 Rho-derived scotopic function. Lineage tracing in mice treated with N-methyl-N-nitrosourea,

11 a disease model of photoreceptor degeneration, showed that the regenerated Rho-positive

12 cells were originated from endogenous MCs. Finally, the regeneration of Rho-positive cells

13 was also induced in the retina of rd10 mice, a model with similar genetic mutation as humans.

14 Notably, the intravitreal injection significantly reduced cone cell death in rd10 mice. This

15 treatment could be a new strategy in retinal regenerative medicine where mammalian

16 endogenous MCs are reprogrammed into photoreceptor cells independent of transplantation

17 or gene transfer. 


\section{Introduction}

2 Various retinal diseases damage photoreceptor cells, leading to irreversible visual

3 dysfunction ${ }^{1,2}$. There are many researches in this decade on induced pluripotent stem cell

4 transplantation and gene therapy to treat the visual dysfunction of retinitis pigmentosa (RP)

5 and age-related macular degeneration (AMD) that are typical retinal degenerative

6 diseases ${ }^{3-5}$. The results of these preclinical and clinical studies revealed that stem cell

$7 \quad$ therapy $^{6}$ and viral gene transfer ${ }^{7}$ are effective in restoring vision. However, these therapies

8 have disadvantages, namely, the high cost of developing and manufacturing cellular

9 medicine; the risk of complications such as immune rejection and tumorigenesis; and the

10 need for special techniques for transplanting cells/cell sheets and vectors ${ }^{8}$.

12 In central nerve regeneration, it has been reported that the administration of small-molecule

13 compounds enables reprogramming into neurons and neural progenitor cells ${ }^{9-11}$. Mahato et

14 al. showed that fibroblasts could differentiate into retinal progenitor cells $\mathbf{s}^{12}$. Zhang et al.

15 confirmed that endogenous astrocytes differentiate into neurons in mice injected with

16 inhibitors into their ventricles ${ }^{13}$. These reports served as motivations to explore the possibility

17 of differentiation of retinal component cells (i.e., endogenous cells) into photoreceptor cells

18 following the administration of compounds. 
2 Retinal glial cells are composed of Müller cells (MCs), astrocytes, and microglia ${ }^{14}$. In

3 zebrafish, MCs have been shown to be a source of new photoreceptor cells after retinal

4 injury ${ }^{15}$. Unfortunately, mammalian MCs do not possess the ability to generate new

5 photoreceptor cells. However, Ueki et al. ${ }^{16}$ and Jorstad et al. ${ }^{17}$ reported that MCs could be

6 reprogrammed into retinal neurons in mice by gene transfer of Ascl1; this gene is essential

7 for photoreceptor regeneration in zebrafish, similar to high mobility group A1 (HMGA1) ${ }^{18}$.

8 Additionally, Yao et al. showed MC-derived rod cell production by gene transfer of $\beta$-catenin,

9 Otx2, Crx, and $\mathrm{Nr}^{19}$. Based on the findings of previous researches, we hypothesized that it

10 might be possible to produce MC-derived photoreceptors by stimulation with small-molecule

11 compounds.

13 In the present study, we demonstrated that the administration of four compounds (SB431542,

14 LDN193189, CHIR99021, and DAPT) could reprogram mouse MCs into rhodopsin (Rho)-

15 positive cells in vitro and in vivo. We showed that intravitreal injection of these compounds

16 induced the differentiation of endogenous MCs into Rho-positive cells in mice treated with N-

17 methyl-N-nitrosourea (MNU) and in rd10 mice; these are disease models of retinal

18 degeneration. Along with the regeneration of Rho-positive cells, the a-wave of the 
1 electroretinogram (ERG) was restored, suggesting that Rho-positive cells had properties

2 similar to those of rod cells.

3

4 In retinal diseases such as RP and AMD, irreversible damage to photoreceptor cells

5 progresses gradually ${ }^{2}, 20$. Unlike cell transplantation and gene therapy, intravitreal injection

6 of compounds is inexpensive and easy to re-perform, and it may be applicable to patients at

$7 \quad$ various stages of the disease.

8

9 Results

10 Screening of small-molecule compounds for converting MCs to photoreceptor cells.

11 We isolated MCs from 6-week-old B6J mice, according to previous reports (Supplementary

12 Fig. 1$)^{21}$. We examined the changes in the expression of photoreceptor markers and

13 transcription factors in primary MCs after stimulation with candidate compounds to test

14 whether the compounds could chemically reprogram MCs into photoreceptor cells.

16 We selected five compounds that act on signaling pathways important for neural

17 differentiation; these have been used in multiple studies $9,10,13,22-26$. The selection is based on

18 previous reports on the successful differentiation of somatic cells, including fibroblasts and 
1 astrocytes, into neurons by small-molecule compounds. The compounds used were the

2 following: SB431542 (tumor growth factor- $\beta$ [TGF- $\beta$ ] inhibitor), LDN193189 (bone

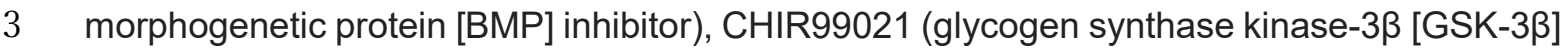

4 inhibitor), DAPT ( $\mathrm{Y}$-secretase inhibitor), and Y-27632 (ROCK inhibitor).

5

6 The time course of the in vitro experiments is shown in Fig. 1a. First, the expression of

7 photoreceptor markers was examined by polymerase chain reaction (PCR) analysis 7 days

8 after stimulation with all 31 combinations of the five compounds (Fig. 1b). The expression

9 level of the messenger RNA (mRNA) of opsin (cone cell marker) was below detection

10 sensitivity in all combinations. On one hand, the expression level of Rho (rod cell marker)

11 mRNA was markedly increased to about 30 -fold of the control in the combination of

12 SB431542, LDN193189, CHIR99021, and DAPT (Fig. 1B). Immunocytochemistry showed

13 that these four compounds induced the expression of Rho protein in $25 \%$ of living cells (Fig.

14 1c, d). The expression levels of genes regulated by these inhibitors were also evaluated.

15 Compound stimulation increased the expression of Axin2 (a downstream molecule of the

16 Wnt/ß-catenin pathway) and decreased the expression of LTBP1 (a downstream molecule

17 of TGF- $\beta$ pathway), Id (a downstream molecule of BMP pathway), and DLL1 (a downstream

18 molecule of Notch pathway), suggesting that the signaling pathways corresponding to each 
compound were inhibited (Supplementary Fig. 2).

2

3 The morphology of the MCs gradually changed from day 4, and a neurite-like structure was

4 observed on day 7 (Fig. 1e). The expression of CD44, an MC marker27, significantly

5 decreased with the change in cell morphology (Fig. 1f). We found that Ascl1 expression

6 peaked on day 1. HMGA1, which is essential for the regeneration of photoreceptor cells in

$7 \quad$ zebrafish $^{16,18}$, was significantly upregulated after stimulation (Supplementary Fig. 3). We also

8 confirmed increased expression of SOX2, a retinal progenitor marker ${ }^{28}, 1$ day after

9 stimulation with the four compounds (Supplementary Fig. 3). In addition, we quantified the

10 expression of retinal neuron markers other than Rho, but none of them increased (Fig. 1g).

11 This phenomenon of increased Rho expression and decreased expression of glial cell

12 markers was observed not only in primary mouse cells, but also in rat cell lines (Fig. 1h).

13 These results suggest that MCs can be differentiated into rod-like cells by the simultaneous

14 administration of the four compounds.

16 Ectopic expression of Rho in wild-type mice by intravitreal injection of compounds.

17 Based on the in vitro results, we explored the possibility that simultaneous administration of

18 these four compounds by intravitreal injection may lead to the differentiation of endogenous 
1 MCs into rod cells. The in vivo experimental protocol is shown in Fig. 2a. Six-week-old wild-

2 type B6J mice (WT mice) received intravitreal injection every 3 days, and Rho expression in

3 the retina was assessed on days 3,7 , and 14 by immunohistochemistry (IHC) and PCR. The

4 layered structure of the retina is shown in Fig. 2b. Although there was no difference in Rho

5 expression in the outer retina between the compound-stimulated and control groups on days

67 and 14, ectopic Rho expression in the outer plexiform layer (OPL) was detected in the

7 compound-stimulated group (Fig. 2c). The mRNA expression levels of retinal neuron-specific

8 markers, including Rho, in the whole retina did not differ between the two groups (Fig. $2 \mathrm{~d}$

9 and Supplementary Fig. 4a). To confirm the induction of Rho expression in MCs by four-

10 compound stimulation, CD44+ CD45- cells were purified from retinas by magnetic-activated

11 cell separation, and Rho mRNA in these cells was quantified by PCR. As shown in Fig. 2e,

12 a significant increase in Rho expression was observed in the compound-stimulated group. It

13 is also noteworthy that there was no increase in other neural markers, except for Rho, in

14 CD44+ CD45- cells (Fig. 2f). The upregulation of Rho expression was only observed on day

153 and may be due to the decrease in the expression of CD44 as MCs differentiated into Rho-

16 positive cells. ERGs showed no clear difference in amplitude of a-wave, b-wave, or oscillatory

17 potential between the control and compound-stimulated groups, and there was no evidence

18 that the newly produced Rho-positive cells functioned as neurons (Supplementary Fig. 4b). 
2 Why was the expression of Rho in WT mice only "ectopic"? Unlike mammals, zebrafish can

3 regenerate the retina. Retinal regeneration occurs when retinal progenitor cells, generated

4 by the migration and division of MCs activated by injury, differentiate into different types of

5 neurons ${ }^{29}$. Ueki et al. reported that overexpression of Ascl1 in MCs causes MCs to

6 differentiate into retinal neurons such as amacrine cells, bipolar cells, and photoreceptor cells,

7 but this regenerative response does not occur without retinal damage ${ }^{16}$. These reports

8 suggest that retinal injury forms a "niche" suitable for regeneration. In addition, adherens

9 junctions and tight junctions are well developed in the outer limiting membrane (OLM), and

10 the permeability of cells and substances is strictly controlled ${ }^{30}$. This barrier may prevent the

11 newly born Rho-positive cells from extending their cell bodies between the OLM and retinal

12 pigment epithelium (RPE).

14 Thus, we hypothesized that injury to the outer retinal layer would create an environment

15 suitable for photoreceptor regeneration. In this study, we used two models of retinal

16 degeneration to confirm the possibility that the four compounds could induce MCs to

17 differentiate into photoreceptor cells. 
1 Induction of Rho expression in the outer retina and restoration of retinal function in MNU-

2 treated mice.

3 First, we used a model of chemical retinal degeneration induced by MNU, that has a potent

4 carcinogen, teratogen, and mutagen ${ }^{31}$. MNU damages the outer retinal layers, such as the

5 RPE layer, outer nuclear layer (ONL), and OPL, while causing little damage to the inner

6 retinal layers. MNU induces progressive photoreceptor apoptosis, RPE degeneration, and

7 subretinal fibrosis; it has been widely used in research to elucidate the pathogenesis of RP

8 and $\mathrm{AMD}^{32}$. Furthermore, MNU promotes $\mathrm{MC}$ hypertrophy and upregulates the expression

9 of glial fibrillary acidic protein (GFAP), a stress marker in MCs that may create an "injury-

10 induced niche" suitable for MCs to differentiate into photoreceptor cells.

12 We determined the dose of MNU at which the ONL disappeared 7 days after intraperitoneal

13 injection (Supplementary Fig. 5). We then performed intravitreal injections of the four

14 compounds on days 0,3 , and 6 after administration of $75 \mathrm{mg} / \mathrm{kg} \mathrm{MNU}$ to investigate whether

15 photoreceptor regeneration occurred in the outer retinal layer (Fig. 3a). The IHC results are

16 shown in Fig. 3b. Peanut agglutinin (PNA) that selectively binds to the cone inner and outer

17 segments, did not differ between the control and compound-stimulated groups; however,

18 Rho expression was significantly increased in the compound-stimulated group (Fig. 3b, c). 
1 Quantification of the percentage of Rho-expressing retinal region in the total retinal length

2 showed that Rho expression was significantly higher after intravitreal injection of the four

3 compounds (Fig. 3d).

4

5 We then tested whether the difference in the expression of Rho-positive cells between the

6 control and compound-stimulated groups depended on cell regeneration or on the inhibition

7 of cell apoptosis. PCR showed a significant increase in Rho mRNA only on day 7 after the

8 administration of four compounds (Fig. 3e and Supplementary Fig. 6), but there was no

9 difference in the number of apoptotic cells (Fig. 3f, g), suggesting that new Rho-positive cells

10 may have been produced.

12 We used a lineage-tracing model that expressed the td-Tomato protein to track MCs, for

13 confirmation that the Rho-positive cells originated from MCs. PAAV.GFAP.Cre.WPRE.hGH,

14 which is an adeno-associated virus (AAV) containing human GFAP promoter, was injected

15 into the vitreous of 2-week-old ROSA-td-Tomato mice so that MCs could be visualized. Four

16 weeks after injection into the vitreous, IHC showed that td-Tomato co-localized with

17 glutamine synthetase, indicating the introduction of AAV into MCs (Supplementary Fig. 7).

18 On day 7 after MNU administration, td-Tomato was co-expressed with Rho in the outer retinal 
1 layer, suggesting that Rho-positive cells originated from MCs (Fig. 3h). Fluorescent-activated

2 cell sorting (FACS) results showed that treatment with the compounds increased the

3 expression level of Rho, but did not change the expression levels of other neural markers in

4 Tomato-positive cells (Fig 3i). These results were consistent with those obtained in primary

5 cells and WT mice, suggesting that td-Tomato-positive MCs were reprogrammed to rods or

6 rod-like cells, not to other retinal neurons, by the four compounds administered. Postsynaptic

7 density protein 95 (PSD95), a synaptic marker, was also expressed around newly produced

8 Rho-positive cells in the OPL of the retina (Fig. 3j).

10 Next, to evaluate whether the four compounds could restore retinal dysfunction, ERGs were

11 recorded 7 days after the four compounds were injected into MNU-treated mice. In mice

12 treated with $75 \mathrm{mg} / \mathrm{kg} \mathrm{MNU}$, the four compounds increased Rho expression in IHC (Fig 3b),

13 but there was no obvious improvement in ERG (Fig 4a). Since the ERG waveform

14 disappeared after the $75 \mathrm{mg} / \mathrm{kg} \mathrm{MNU}$ administration, it was possible that the retinal

15 dysfunction might have been so severe that the effect of the four compounds could not be

16 detected. Therefore, the dose was reduced to $30 \mathrm{mg} / \mathrm{kg}$ and ERG was performed again. As

17 shown in Fig. 4a and 4b, the amplitude of the a-waves was significantly improved by the

18 injection of the four compounds. These findings suggest that Rho-positive cells induced by 
1 simultaneous administration of the four compounds to the vitreous restored retinal

2 dysfunction in MNU-treated mice.

4 Induction of Rho expression in the outer retina and inhibition of cone cell death in rd10 mice.

5 Finally, we tested the retinal regenerative capacity of these four compounds in rd10 mice, a

6 mouse model of RP with a missense point mutation in the $\beta$-subunit of the rod cyclic

7 guanosine monophosphate phosphodiesterase gene (PDE6 $\beta)^{33}$. Point mutations of PDE6 $\beta$

8 have been detected in patients with autosomal recessive RP ${ }^{34}$. In rd10 mice, rod cell death

9 peaked at 3-4 weeks, followed by cone cell death ${ }^{35}$. We then performed intravitreal injections

10 of the four compounds every 3 days in 4-week-old rd10 mice and investigated the changes

11 in Rho expression (Fig. 5a). We first quantified the expression of retinal neuron markers in

12 the whole retina using PCR. After treatment with the four compounds, only the expression of

13 Rho was upregulated, and the expression levels of other markers remained unchanged. (Fig.

$145 \mathrm{~b}$ and Supplementary Fig. 8). Moreover, IHC showed an obvious increase in Rho

15 expression in the outer retina of the compound-stimulated group (Fig. 5c, d). We quantified

16 the percentage of Rho-expressing retinal region in the total retinal length; indeed, Rho

17 expression was significantly increased by intravitreal injection of the four compounds (Fig.

$185 e)$. 
2 It is known that rod cell death is followed by cone cell death in RP, but the cause of cone cell

3 death is not clearly understood. In addition to the oxidative stress caused by excessive

4 oxygen accumulation in the outer retinal layer due to rod cell death and the release of toxic

5 substances from damaged rod cells ${ }^{33,36}$, Léveillard et al. and Mohand-Said et al. reported

6 that a decrease in the secretion of survival factors from rods is the cause of cone cell

7 death ${ }^{37,38}$. Byrne et al. have shown that cone cell death can be suppressed by gene transfer

8 of the rod-derived cone viability factor (RdCVF) encoded by nucleoredoxin-like 1 (NXNL1) ${ }^{39}$.

10 Next, we counted PNA-positive cone cells based on our previous report ${ }^{33}$ to verify the

11 inhibition of cone cell death associated with increased expression of Rho-positive cells.

12 Retinal whole-mount staining showed that significantly more PNA-positive cone cells were

13 retained on day 7 after intraocular injection of the four compounds (Fig. 5f, g). In addition,

14 the expression of NXNL1 was significantly increased in the compound-stimulated group (Fig.

$155 \mathrm{~h}$ ), suggesting that the newly produced Rho-positive cells may secrete RdCVF.

17 Discussion

18 In this study, we reprogrammed MCs into Rho-positive cells both in vitro and in vivo. These 
1 Rho-positive cells can improve retinal function in a model of retinal degeneration. It should

2 be emphasized that endogenous cells in the retina were only differentiated into

3 photoreceptor-like cells through the simultaneous administration of the four compounds.

5 In WT and MNU-treated mice, we found that MCs were reprogrammed into Rho-positive cells

6 after reverting to progenitor cells by stimulation with the four compounds. MC-driven Rho-

7 positive cells did not show elevated expression of other retinal neural markers, suggesting

8 that MCs could only be induced into rod or rod-like cells. In zebrafish, MC-derived progenitor

9 cells have the ability to differentiate into various types of neurons, but they generate

10 unneeded neurons because of their multipotency ${ }^{40}$. Ueki et al. also reported that MCs

11 overexpressing Ascl1 differentiate not only into photoreceptor cells, but also into amacrine

12 and bipolar cells ${ }^{16}$. If these compounds can be administered to cause MCs to differentiate

13 only into rods or rod-like cells, this therapy will have an advantage over other therapies in

14 terms of safety. MCs play an important role in supporting the function, metabolism, and

15 structure of neurons in the retina. In other words, if MCs are depleted by inducing MCs into

16 nerves, abnormalities in retinal function may occur. However, in this study, there was no

17 obvious abnormality in the retinal layer structure or loss of function due to compound

18 administration. MCs are capable of asymmetric self-renewal after injury ${ }^{41,42}$; therefore, no 
obvious depletion may have occurred.

2

3 This treatment strategy is also valuable in real-world clinical applications. Advances in omics

4 analysis are rapidly revealing the signaling pathways required for neural regeneration.

5 Although still in the preclinical stage, it is now possible to differentiate non-neuronal cells into

6 retinal neurons or retinal progenitor cells by modifying specific signaling pathways through

7 genome editing with virus vectors or compound stimulation. If autologous transplantation is

8 possible, concerns about rejection will be alleviated. However, the development of gene

9 therapy and cell therapy products is costly. Therefore, we decided to investigate the

10 possibility of direct differentiation of retinal endogenous cells into photoreceptor cells using

11 molecular compounds that can be made inexpensively; these compounds have the potential

12 for mass production. From an ophthalmologist's perspective, we also wanted to make the

13 treatment as simple as possible. It has been reported that different signals are required for

14 different stages of neural differentiation, but we wanted to achieve retinal regeneration with

15 a single mixture of molecular compounds.

17 In the mammalian retina, unlike in the central nervous system, there are no reports of neural

18 regeneration solely by direct reprogramming using compounds. However, the signaling 
1 pathways that are modified by the compounds used in this study have also been shown to

2 be important in retinal regeneration. Osakada et al. demonstrated that activation of Wnt/B-

3 catenin signaling by GSK-3 $\beta$ inhibitors promotes the proliferation of MC-derived retinal

4 progenitors after injury ${ }^{43}$. Lenkowski et al. ${ }^{44}$ and Todd et al. $4^{5}$ reported that the TGFb-

5 Smad2/3 signaling pathway regulates the regeneration of retinal neurons after injury in

6 zebrafish. They showed that the inhibition of this pathway suppresses MC-derived gliosis

7 and induces proliferation of MC-derived progenitor cells. In addition, Ueki et al. showed that

8 the activated BMP-Smad1/5/8 signaling pathway promotes MC-derived gliosis in proliferative

9 vitreoretinopathy ${ }^{46}$. Moreover, DAPT increased the expression of Crx, which is important for

10 rod photoreceptor maturation and promotes photoreceptor differentiation. Therefore, we

11 speculate that CHIR99021, a Wnt/ $\beta$-catenin activator, SB431542, a TGF- $\beta$ inhibitor, and

12 LDN193189, a BMP inhibitor, may lead to the proliferation of MC-derived neural progenitor

13 cells and DAPT. A notch inhibitor may contribute to the differentiation toward rod

14 photoreceptors.

16 In vitro results showed that simultaneous administration of these four compounds increased

17 the efficiency of Rho-positive cell production. We confirmed that each targeted signaling

18 pathway was properly inhibited, even when four compounds were administered 
1 simultaneously. The number of Rho-positive cells decreased in the absence of any of the

2 four compounds and with the addition of an extra compound. These results suggest that the

3 suppression of TGF, BMP, and Notch signaling pathways and the activation of the Wnt/ $\mathrm{B}$ -

4 catenin signaling pathway may have created an environment suitable for the regeneration of

5 rod or rod-like cells. In the present study, the efficiency of differentiation of primary MCs into

6 Rho-positive cells was only $25 \%$. Single-cell analysis ${ }^{47,48}$ has shown that there is diversity in

7 MCs, and it is possible that some types are more likely to differentiate into Rho-positive cells

8 upon stimulation with compounds. In addition, in most previous reports, when multiple

9 compounds were used to increase the efficiency of differentiation of non-neuronal cells into

10 neuronal cells, the timing of administration of each compound was changed. When multiple

11 compounds were administered simultaneously, as in the present study, it may have been

12 difficult to strictly control the signals.

14 It was also possible to suppress the apoptosis of cone cells in rd10 mice; this reveals a clinical

15 application of the intravitreal injection of these compounds. The rods are sensitive enough to

16 detect a single photon and are suitable for night vision. However, rods rarely function in bright

17 areas because they are saturated by moderately bright light. On the other hand, the cones

18 are 100 times less sensitive to light than the rods, so they do not function in the dark. However, 
1 the cones have an excellent ability to adjust their sensitivity, so they do not saturate even in

2 bright areas. Generally, visual acuity and color vision depend on the function of the cones ${ }^{36}$.

3 This study suggests that the secretion of RdCVF from MC-derived Rho-positive cells may

4 inhibit the death of cone cells. If the cone cells of RP patients can be maintained, the quality

5 of vision will be improved. The rate of degeneration is slower in human RP as compared to

6 rd10 mice, allowing more time for therapeutic intervention and the ability to treat more

7 patients.

9 The limitation of this study is that the two mouse models, MNU-treated mice and rd10 mice,

10 do not fully reflect human RP and AMD. MNU treatment results in the destruction of the outer

11 retinal layer in approximately one week. In other words, there is a difference between the phenomena that occur in the retinas of MNU-treated mice and those of slowly progressing

13 human RP and AMD. In addition, although rd10 mice have the same mutation as in humans,

14 the same results may not be obtained for all types of RP because more than 100 different

15 causative genes have been reported in human RP. In addition, once-every-three-day

16 intravitreal injections cannot be used in clinical practice. Appropriate dosages and intervals

17 must be established using drug delivery systems or developing extended-release agents. 
1 In this study, we showed that the simultaneous administration of four compounds alone can

2 differentiate retinal MCs into Rho-positive cells and restore the function of the injured retina.

3 This method can be applied not only to RP and AMD, but also to photoreceptor degeneration

4 caused by various retinal diseases. Vitreous injection of the compound is expected to be a

5 new strategy for retinal regeneration therapy that does not rely on cell transplantation or gene

6 transfer.

7

8 Methods

9 Animals. Male and female B6 wild-type (WT) mice, B6.Cg-Gt(ROSA)26Sortm14(CAG-

10 tdTomato)Hze/J (ROSA-td-Tomato) mice, and B6.CXB1-Pde6ßrd10/J (rd10) mice were used.

11 ROSA-td-Tomato and rd10 mice were purchased from the Jackson Laboratory. To perform

12 the experiments, mice were anesthetized by intraperitoneal injection of $15 \mathrm{mg} / \mathrm{kg}$ ketamine

13 and $7 \mathrm{mg} / \mathrm{kg}$ xylazine. The chemical-induced retinal degeneration model was created by

14 intraperitoneal administration of a single dose of MNU in mice. After anesthesia, the mice

15 were weighed, and MNU was injected (30-75 mg/kg). All mice were treated in accordance

16 with the standards of the Association for Research in Vision and Ophthalmology for the use

17 of animals in ophthalmic and vision research. All animal experiments were reviewed and

18 approved by the Kyushu University Ethics Committee for Animal Experimentation and were 
1 conducted in accordance with the relevant guidelines and regulations (A20-360, A21-225).

3 Cell culture. Primary retinal MCs were cultured as previously described ${ }^{21}$. Briefly, the eyes

4 of 6-week-old WT mice were enucleated under sterile conditions. Isolated retinas were then

5 digested with collagenase $\mathrm{D}(1.2 \mathrm{mg} / \mathrm{mL}$, Roche, Mannheim, Germany) and DNAse I

$6 \quad(0.005 \%$, Roche). Cells were seeded onto a Poly L-Lysine-coated dish (Corning, NY, USA)

7 and cultured at $34^{\circ} \mathrm{C}$ in $5 \% \mathrm{CO}_{2}$. The culture medium (CM) was composed of DMEM high

8 glucose (Sigma-Aldrich, St Louis, MO, USA), 0.1\% penicillin-streptomycin, and $10 \%$ fetal

9 bovine serum. The rat MC cell line (TR-MUL5) was purchased from Fact, Inc. (Sendai, Japan),

10 and cultured in the same culture medium.

12 Stimulation with small-molecule compounds in MCs and TR-MUL5 cells in vitro. One

13 day before administration of the compounds, half of the culture medium was replaced with

14 the stimulating medium composed of Ham's F-12 (FUJIFILM Wako, Osaka, Japan), N2

15 supplement (Thermo, Waltham, MA, USA), and $0.1 \%$ penicillin-streptomycin. The next day,

16 all of the medium was replaced with stimulating medium. Five compounds, namely,

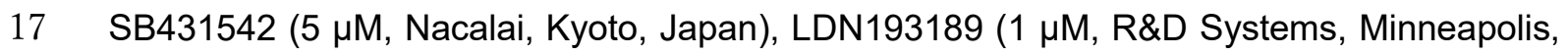

18 MN, USA), CHIR99021 (1.5 $\mu$ M Cayman, Ann Arbor, MI, USA), DAPT (5 $\mu$ M, Sigma-Aldrich), 
1 and Y-27632 (0.5 $\mu \mathrm{M}$, Nacalai) were added to the SM. The medium was changed every two

2 days and the compounds were added at the same time.

4 Intravitreal injection. After anesthesia, the compounds were injected into the vitreous of 6-

5 week-old WT, ROSA-td-Tomato, and 2-and 4-week-old rd10 mice under topical mydriasis.

6 The final concentrations of SB431542, LDN193189, CHIR99021, and DAPT were $0.1 \mu \mathrm{M}$,

$7 \quad 0.02 \mu \mathrm{M}, 0.03$, and $0.1 \mu \mathrm{M}$, respectively. Micro syringes with 33-gauge needles (Hamilton,

8 Reno, NV, USA) were used. After anesthesia, we injected intravitreally of $1 \mu \mathrm{l}$

9 pAAV.GFAP.Cre.WPRE.hGH, with a concentration was $7 \times 10^{13} \mathrm{vg} / \mu \mathrm{L}$, to 2-week-old ROSA-

10 td-Tomato mice. PAAV.GFAP.Cre.WPRE.hGH was a gift from James M. Wilson (Addgene

11 plasmid \# 105550 ; http://n2t.net/addgene:105550 ; RRID:Addgene_105550).

13 Quantitative real-time PCR (qPCR). Total RNA of retinal tissue and cultured cells was extracted using the NucleoSpin RNA Kit (Macherey-Nagel, Düren, Germany) according to the manufacturer's instructions. RNA was reverse transcribed using the First Strand cDNA

16 Synthesis Kit for RT-PCR (Roche). Real-time qPCR was performed using SYBR Premix Ex

17 Taq (Takara, Shiga, Japan) with a LightCycler 96 (Roche). The primer sequences used are

18 listed in Supplementary Table 1. 
2 Immunocytochemistry. Cultured cells were fixed with $4 \%$ paraformaldehyde at room

3 temperature for $20 \mathrm{~min}$. After washing with phosphate buffered saline (PBS), cells were

4 incubated with $0.3 \%$ Triton-X 100 (FUJIFILM Wako) in PBS for 15 min and blocked with PBS

5 containing $10 \%$ normal goat serum (NGS) (Thermo) for $1 \mathrm{~h}$ at room temperature. Cells were

6 then incubated with primary antibodies (chicken anti-vimentin [ab24525, Abcam, Cambridge,

7 UK], rabbit anti-glutamine synthetase [GS] [ab73593, Abcam], mouse anti-rhodopsin [1D4,

8 Abcam], and rat anti-mouse CD44-PE [1M7, eBioscience, San Diego, CA, USA]) at $4^{\circ} \mathrm{C}$

9 overnight. Next, the cells were incubated with Alexa-conjugated secondary antibodies

10 (Thermo) for $1 \mathrm{~h}$ at room temperature, and the nuclei were counterstained with 4',6-

11 diamidino-2-phenylindole (DAPI). Immunofluorescence images were acquired using a BZ-

12 X710 microscope (Keyence, Osaka, Japan). For cell counting, at least three locations (fields)

13 were selected per dish.

Immunohistochemistry (frozen section). Eyes were enucleated, fixed with 4\%

16 paraformaldehyde for $30 \mathrm{~min}$ at room temperature, and immersed in OCT compound. Frozen

17 sections were cut with a cryostat (CM1800, Leica Microsystems, Wetzlar, Germany) at $8 \mu \mathrm{m}$

18 and mounted onto MAS-coated glass slides (MAS-01, Matsunami, Osaka, Japan). After 
1 removing the OCT compound in PBS containing $0.3 \%$ Triton $\mathrm{X}-100$ for $15 \mathrm{~min}$, sections were

2 blocked with $10 \%$ NGS in PBS for $1 \mathrm{~h}$. Sections were then incubated with primary antibodies

3 (chicken anti-vimentin [ab24525, Abcam], mouse anti-rhodopsin [1D4, Abcam], FITC-

4 conjugated PNA [L7381, Sigma-Aldrich], rabbit anti-PSD95 [D27E11, Cell Signaling

5 Technology, Danvers, MA, USA], rabbit anti-GS [ab73593, Abcam]) at $4^{\circ} \mathrm{C}$ overnight. After

6 washing with PBS, the sections were incubated with Alexa-conjugated secondary antibody

7 for $1 \mathrm{~h}$ at room temperature, and the nuclei were counterstained with DAPI.

8

9 Immunohistochemistry (paraffin section). After fixation with 4\% paraformaldehyde for 30

$10 \mathrm{~min}$ at room temperature, the eyes of mice were embedded in paraffin and sliced into $3-\mu \mathrm{m}$

11 sections. After removal of the paraffin, the sections were activated, blocked, and incubated

12 with primary antibodies (chicken anti-vimentin [ab24525, Abcam], mouse anti-rhodopsin

13 [1D4, Abcam], FITC-conjugated PNA [L7381, Sigma-Aldrich], rabbit anti-PSD95 [D27E11,

14 Cell Signaling Technology, rabbit anti-GS [ab73593, Abcam]) at $4{ }^{\circ} \mathrm{C}$ overnight. After washing

15 with PBS, the sections were incubated with Alexa-conjugated secondary antibody for $1 \mathrm{~h}$ at

16 room temperature, and the nuclei were counterstained with DAPI. Images were acquired

17 using a BZ-X710 microscope (Keyence). Rho-positive length in the outer retinal layers was

18 analyzed using ImageJ software. At least three sections, including the optic nerve, were 
1 measured per eye, and the average was calculated.

3 Fluorescent activated cell sorting (FACS). For flow cytometry measurements, cultured

4 cells were washed with PBS, $0.25 \%$ trypsin was added, and the cells were incubated for 3

$5 \min$ at $37^{\circ} \mathrm{C}$. After washing with $2 \%$ bovine serum albumin (BSA) in PBS, the cells were fixed

6 with $1 \%$ paraformaldehyde and permeabilized in $0.3 \%$ Triton $\mathrm{X}-100$ for 10 min at $4{ }^{\circ} \mathrm{C}$. After

7 rinsing, the cells were incubated with an antibody (rat anti-Vimentin-APC [IC2105A, R\&D

8 Systems], rat anti CD44-PE [eBioscience]) for $1 \mathrm{~h}$ at $4^{\circ} \mathrm{C}$. After rinsing, the cells were

9 resuspended in 2\% BSA in PBS and analyzed by FACS. FACSVerse and FlowJo software

10 (BD Biosciences, Franklin Lakes, NJ, USA) were used for the analysis. For td-Tomato-

11 positive cell sorting, isolated retinas from ROSA-td-Tomato mice were incubated with

12 collagenase $\mathrm{D}(1.2 \mathrm{mg} / \mathrm{ml})$ and DNAse I $(0.005 \%)$ for $20 \mathrm{~min}$ at $37^{\circ} \mathrm{C}$. After washing with $2 \%$

13 BSA in PBS, the cells were stained with 7-Amino-Actinomycin D Viability Staining Solution

14 (BioLegend, San Diego, CA, USA) to remove dead cells. A BD FACSAria ${ }^{\text {TM }}$ III cell sorter (BD

15 Biosciences) was used for live td-Tomato-positive cell sorting.

17 TUNEL staining. TUNEL staining was performed using an ApopTag Fluorescein In Situ

18 Apoptosis Detection Kit (Merck Millipore, Darmstadt, Germany) according to the 
1 manufacturer's instructions. The number of TUNEL-positive cells in the ONL was counted as

2 described for MNU-treated mice ${ }^{49}$. Briefly, the number of TUNEL-positive cells in the ONL of

3 a $9000(150 \times 60) \mu m^{2}$ area, each $400 \mu \mathrm{m}$ away from the optic nerve papilla and ciliary body,

4 were counted in a masked fashion. Then, we calculated the average number of TUNEL-

5 positive cells in four areas per section.

6

7 Retinal whole-mount staining. Eyes were enucleated and fixed with $4 \%$

8 paraformaldehyde for $1 \mathrm{~h}$ at $4^{\circ} \mathrm{C}$. The isolated retinas were incubated with PBS containing

$9 \quad 0.5 \%$ Triton-X 100 for 30 min and blocked for $1 \mathrm{~h}$ with PBS containing 10\% NGS and $0.5 \%$

10 Triton-X 100. The retinas were then incubated with FITC-conjugated PNA (L7381, Sigma-

11 Aldrich) at $4^{\circ} \mathrm{C}$ overnight. After washing with PBS, the retinas were mounted on slides. To

12 assess the number of cone photoreceptor cells, we counted the PNA-positive cells in $125 \times$

$13125 \mu \mathrm{m}^{2}$ retinal areas in the superior, inferior, temporal, and nasal areas located $250 \mu \mathrm{m}$,

$14500 \mu \mathrm{m}$, and $1,000 \mu \mathrm{m}$ from the optic disc. The names and conditions of the samples were

15 masked by the observers. The number of PNA-positive cells was averaged from 12 retinal

16 areas in each retina.

17 Immunofluorescence images were acquired using a BZ-X710 microscope (Keyence). 
1 Electroretinograms. ERGs were recorded as described in detail ${ }^{50}$. Briefly, mice were dark-

2 adapted overnight and anesthetized under dim red light. After mydriasis, recordings were

3 made using the PuREC system (Mayo, Aichi, Japan), as previously reported ${ }^{51}$. ERGs were

4 recorded on day 7 after intravitreal injections of the four compounds, according to the

5 International Society for Clinical Electrophysiology of Vision standard protocol with five light

6 stimuli per recording. The scotopic ERGs were elicited using a stimulus intensity of

$7 \quad 10000 \mathrm{~cd} \mathrm{~m}^{-2}$. The responses were differentially amplified and filtered between 0.3 and

$8500 \mathrm{~Hz}$

10 Statistics and reproducibility. Data are presented as the mean \pm standard error of mean

11 (SEM). Statistical analysis was performed using JMP Pro version 16.0 (SAS Institute). The

12 significance of the difference was determined using two-tailed Student's $t$-test. Differences

13 were considered statistically significant at $P<0.05$. The number of biological repeats $(\mathrm{n})$ for

14 each experiment is noted in the figure legends. 


\section{References}

2 1. Busskamp, V., Picaud, S., Sahel, J. A., \& Roska, B. Optogenetic therapy for retinitis

3 pigmentosa. Gene Ther. 19, 169-175 (2012).

4 2. Hartong, D. T., Berson, E. L., \& Dryja, T. P. Retinitis pigmentosa. Lancet 368, 1795-1809

5 (2006).

6 3. Kuriyan, A. E. et al. Vision Loss after Intravitreal Injection of Autologous "Stem Cells" for

$7 \quad$ AMD. N. Engl. J. Med. 376, 1047-1053 (2017).

8 4. Mazzilli, J. L. et al. A preclinical safety study of human embryonic stem cell-derived retinal

9 pigment epithelial cells for macular degeneration. J. Ocul. Pharmacol. Ther. 36, 65-69 (2020).

10 5. Sanges, D. et al. Reprogramming Müller glia via in vivo cell fusion regenerates murine

11 photoreceptors. J. Clin. Invest. 126, 3104-3116 (2016).

12 6. Mandai, M. et al. Autologous induced stem-cell-derived retinal cells for macular

13 degeneration. N. Engl. J. Med. 376, 1038-1046 (2017).

14 7. Cehajic-Kapetanovic, J. et al. Initial results from a first-in-human gene therapy trial on X-

15 linked retinitis pigmentosa caused by mutations in RPGR. Nat. Med. 26, 354-359 (2020).

16 8. Wright, L. S. et al. Induced pluripotent stem cells as custom therapeutics for retinal repair:

17 progress and rationale. Exp. Eye Res. 123, 161-172 (2014).

18 9. Hu, W. et al. Direct conversion of normal and Alzheimer's disease human fibroblasts into 
1 neuronal cells by small molecules. Cell Stem Cell. 17, 204-212 (2015).

2 10. $\mathrm{Li}, \mathrm{X}$. et al. Small-molecule-driven direct reprogramming of mouse fibroblasts into

3 functional neurons. Cell Stem Cell. 17, 195-203 (2015).

4 11. Cheng, L. et al. Generation of neural progenitor cells by chemical cocktails and hypoxia.

5 Cell Res. 25, 645-646 (2015).

6 12. Mahato, B. et al. Pharmacologic fibroblast reprogramming into photoreceptors restores

7 vision. Nature 581, 83-88 (2020).

8 13. Zhang, L. et al. Small molecules efficiently reprogram human astroglial cells into

9 functional neurons. Cell Stem Cell. 17, 735-747 (2015).

10 14. Vecino, E. et al. Glia-neuron interactions in the mammalian retina. Prog. Retin. Eye Res.

$115 \mathbf{5 1}, 1-40(2016)$.

12 15. Goldman D. Müller glial cell reprogramming and retina regeneration. Nat. Rev. Neurosci.

$13 \quad 15,431-442(2014)$.

14 16. Ueki, Y. et al. Transgenic expression of the proneural transcription factor Ascl1 in Müller

15 glia stimulates retinal regeneration in young mice. PNAS 112, 13717-13722 (2015).

16 17. Jorstad, N. L. et al. Stimulation of functional neuronal regeneration from Müller glia in

17 adult mice. Nature 548, 103-107 (2017).

18 18. Hoang, T. et al. Gene regulatory networks controlling vertebrate retinal regeneration. 
1 Science $\mathbf{3 7 0}$, eabb8598 (2020).

2 19. Yao, K. et al. Restoration of vision after de novo genesis of rod photoreceptors in

3 mammalian retinas. Nature 560, 484-488 (2018).

4 20. Curcio, C. A., Medeiros, N. E., \& Millican, C. L. Photoreceptor loss in age-related macular

5 degeneration. Invest. Ophthalmol. Vis. Sci. 37, 1236-1249 (1996).

6 21. Hicks, D., \& Courtois, Y. The growth and behaviour of rat retinal Müller cells in vitro. 1.

$7 \quad$ An improved method for isolation and culture. Exp. Eye Res. 51, 119-129 (1990).

8 22. Dai, P., Harada, Y., \& Takamatsu, T. Highly efficient direct conversion of human fibroblasts

9 to neuronal cells by chemical compounds. J. Clin. Biochem. Nutr. 56, 166-170 (2015).

10 23. Shi, Y. et al. Induction of pluripotent stem cells from mouse embryonic fibroblasts by Oct4

11 and Klf4 with small-molecule compounds. Cell Stem Cell. 3, 568-574 (2008).

12 24. Wang, Y. et al. Chemical conversion of mouse fibroblasts into functional dopaminergic

13 neurons. Exp. Cell Res. 347, 283-292 (2016).

14 25. Cheng, L. et al. Direct conversion of astrocytes into neuronal cells by drug cocktail. Cell Res. 25, 1269-1272 (2015).

16 26. Gao, L. et al. Direct generation of human neuronal cells from adult astrocytes by small

17 molecules. Stem Cell Reports 8, 538-547 (2017).

18 27. Wang, H. et al. Short hairpin RNA-mediated knockdown of VEGFA in Müller cells reduces 
1 intravitreal neovascularization in a rat model of retinopathy of prematurity. Am. J. Pathol. 183,

$2964-974(2013)$.

3 28. Taranova, O. V. et al. SOX2 is a dose-dependent regulator of retinal neural progenitor

4 competence. Genes Dev. 20, 1187-1202 (2006).

5 29. Wan, J., \& Goldman, D. Retina regeneration in zebrafish. Curr. Opin. Genet. Dev. 40,

$6 \quad 41-47(2016)$.

7 30. Omri, S. et al. The outer limiting membrane (OLM) revisited: clinical implications. Clin.

8 Ophthalmol. 4, 183-195 (2010).

9 31. Tsubura, A., Yoshizawa, K., Kuwata, M., \& Uehara, N. Animal models for retinitis

10 pigmentosa induced by MNU; disease progression, mechanisms and therapeutic trials. Histol.

11 Histopathol. 25, 933-944 (2010).

12 32. Chen, Y., Luo, X., Liu, S., \& Shen, Y. Neuroprotective effect of cannabinoid receptor 1

13 antagonist in the MNU-induced retinal degeneration model. Exp. Eye Res. 167, 145-151

14 (2018).

15 33. Nakatake, S. et al. MUTYH promotes oxidative microglial activation and inherited retinal

16 degeneration. JCI Insight 1, e87781 (2016).

17 34. McLaughlin, M. E., Sandberg, M. A., Berson, E. L., \& Dryja, T. P. Recessive mutations in

18 the gene encoding the $\beta$-subunit of rod phosphodiesterase in patients with retinitis 
1 pigmentosa. Nat. Genet. 4, 130-134 (1993).

2 35. Kanan, Y. et al. Metipranolol promotes structure and function of retinal photoreceptors in

3 the rd10 mouse model of human retinitis pigmentosa. J. Neurochem. 148, 307-318 (2019).

4 36. Campochiaro, P. A., \& Mir, T. A. The mechanism of cone cell death in retinitis pigmentosa.

5 Prog. Retin. Eye Res. 62, 24-37 (2018).

6 37. Léveillard, T. et al. Identification and characterization of rod-derived cone viability factor.

$7 \quad$ Nat. Genet. 36, 755-759 (2004).

8 38. Mohand-Said, S. et al. Normal retina releases a diffusible factor stimulating cone survival

9 in the retinal degeneration mouse. PNAS 95, 8357-8362 (1998).

10 39. Byrne, L. C. et al. Viral-mediated RdCVF and RdCVFL expression protects cone and rod

11 photoreceptors in retinal degeneration. J. Clin. Invest. 125, 105-116 (2015).

12 40. Powell, C. et al. Zebrafish Müller glia-derived progenitors are multipotent, exhibit

13 proliferative biases and regenerate excess neurons. Sci. Rep. 6, 24851 (2016).

14 41. Kassen, S. C. et al. Time course analysis of gene expression during light-induced

15 photoreceptor cell death and regeneration in albino zebrafish. Dev. Neurobiol. 67, 1009-1031

16 (2007).

17 42. Nagashima, M., Barthel, L. K., \& Raymond, P. A. A self-renewing division of zebrafish

18 Müller glial cells generates neuronal progenitors that require $\mathrm{N}$-cadherin to regenerate retinal 
neurons. Development 140, 4510-4521 (2013).

2 43. Osakada, F. et al. Wnt signaling promotes regeneration in the retina of adult mammals.

3 J. Neurosci. 27, 4210-4219 (2007).

4 44. Lenkowski, J. R. et al. Retinal regeneration in adult zebrafish requires regulation of TGF $\beta$

$5 \quad$ signaling. Glia 61, 1687-1697 (2013).

6 45. Todd, L. et al. BMP- and TGFß-signaling regulate the formation of Müller glia-derived

7 progenitor cells in the avian retina. Glia 65, 1640-1655 (2017).

8 46. Ueki, Y., \& Reh, T. A. EGF stimulates Müller glial proliferation via a BMP-dependent

9 mechanism. Glia 61, 778-789 (2013).

10 47. Hu, F. Y. et al. Cell development deficiency and gene expression dysregulation of trisomy

1121 retina revealed by single-nucleus RNA sequencing. Front. Bioeng. Biotechnol. 8, 564057

12 (2020).

13 48. Menon, M. et al. Single-cell transcriptomic atlas of the human retina identifies cell types

14 associated with age-related macular degeneration. Nat. Commun. 10, 4902 (2019).

49. Yoshizawa, K. et al. Caspase-3 inhibitor rescues N-methyl-N-nitrosourea-induced retinal

16 degeneration in Sprague-Dawley rats. Exp. Eye Res. 71, 629-635 (2000).

17 50. Tanimoto, N. et al. Vision tests in the mouse: Functional phenotyping with

18 electroretinography. Front. Biosci. (Landmark Ed.) 14, 2730-2737 (2009). 
1 51. Nakama, T. et al. Inhibition of choroidal fibrovascular membrane formation by new class

2 of RNA interference therapeutic agent targeting periostin. Gene Ther. 22, 127-137 (2015). 


\section{Acknowledgements}

2 The authors thank lori Wada, Mitsuhiro Kurata, Masayo Eto, and Fumiyo Morikawa (Kyushu

3 University) for technical assistance. This study was supported by JSPS KAKENHI with grant

4 numbers JP18H02956 and JP21H03094, given to KH Sonoda. Research funding was from

5 Senju Pharmaceutical Co., Ltd., given to M Arima, Y Murakami, and KH Sonnoda. The

6 funders had no role in the study design, data collection and analysis, decision to publish, or

7 preparation of the manuscript. The authors also thank Editage (www.editage.com) for English

8 language editing.

10 Author contributions

11 M Arima designed this study with $Y$ Fujii. Critical assistance was provided by $Y$ Murakami and $\mathrm{KH}$ Sonoda. Y Fujii and M Arima performed the in vitro and in vivo experiments and data

13 analysis. Y Fujii and M Arima wrote the manuscript. All the authors approved the final version

14 of the manuscript.

\section{Competing Interests}

17 The authors have declared that no conflict of interest exists. 
Figure Legends

2 Figure 1. Screening of small-molecule compounds for converting primary MCs to

3 photoreceptor-like cells.

4 a Time course of in vitro experiments. MCs were stimulated with four compounds every 2

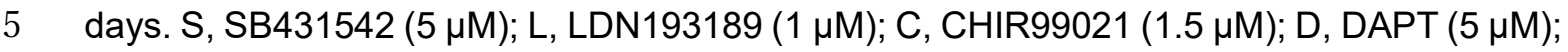

$6 \mathrm{Y}, \mathrm{Y}-27632(0.5 \mu \mathrm{M})$. b Comparison of Rho mRNA expression levels in all 31 combinations

7 using real time qPCR. In the four-compound (SB431542, LDN193189, CHIR99021, DAPT)

8 group, the expression increased about 30-fold compared to the dimethyl sulfoxide (DMSO)

9 group. $\mathrm{n}=3$. $\mathbf{c}$ and $\mathbf{d}$ Immunofluorescence of Rho (c) and the corresponding quantitative

10 results (d) on day 7 after stimulation with the four compounds. c Rho expression increased

11 after the stimulation with four compounds. Scale bar $=100 \mu \mathrm{m}$. d The percentage of Rho

12 positive cells significantly increased after the administration of four compounds. $n=3$. e

13 Microscopic images of cultured cells. A neurite-like structure could be observed on day 7

14 after the stimulation with four compounds. Scale bar $=100 \mu \mathrm{m} . \mathbf{f}$ Real time qPCR results of

15 retinal cell-specific markers and CD44 on day 7 after the stimulation with four compounds.

16 Rho expression significantly increased and CD44 expression significantly decreased by the

17 administration of four compounds. $\mathrm{n}=3$. $\mathbf{g}$ Real time $\mathrm{qPCR}$ results of Rho and glutamine

18 synthetase (GS) expression in TR-MUL5. Rho expression significantly increased on day 1 
1 and 3, and GS expression significantly decreased on day 5 after the stimulation with four

2 compounds. $\mathrm{n}=3$ DMSO group; $\mathrm{n}=5$ compound group.

3 All data are presented as the mean \pm SEM. ${ }^{*} P<0.05,{ }^{* *} P<0.01$, Student's $t$-test.

5 Figure 2. Ectopic expression of Rho in WT mice by intravitreal injection of four

6 compounds.

7 a Time course of in vivo experiments using WT mice. Four compounds were intravitreally

8 injected every 3 days. b Hematoxylin and eosin staining of mouse retina. INL, inner nuclear

9 layer; OPL, outer plexiform layer; ONL, outer nuclear layer; OLM, outer limiting membrane;

10 RPE, retinal pigment epithelium. c Immunohistochemistry of vimentin (green) and Rho (red)

11 on day 7 and 14 after intravitreal injection of compounds. Ectopic Rho (yellow arrows) in OPL

12 appeared after the injection of compounds. Vim, vimentin. Scale bar $=100 \mu \mathrm{m}$. $\mathbf{d}$ Real-time

13 qPCR results of Rho expression. There was no significant change in the level of Rho mRNA

14 in the whole retina $(n=4)$, but it was significantly increased in CD44+CD45- cells. $n=6$. e

15 Real-time qPCR results of retinal neuron specific markers in CD44+ CD45- cells. $n=6$. All

16 data are presented as the mean \pm SEM, ${ }^{*} P<0.05$, Student's $t$-test.

18 Figure 3. Reprograming of MCs into Rho-positive cells in MNU-treated mice. 
1 a Time course of in vivo experiments using MNU-treated mice. After a single systemic

2 administration of $75 \mathrm{mg} / \mathrm{kg} \mathrm{MNU}$, intravitreal injection of four compounds was performed

3 every 3 days. i.p., intraperitoneal injection; TUNEL, TdT-mediated dUTP nick end labeling. b

4 Immunohistochemistry of Rho (yellow arrows) on day 3 and 7 after intravitreal injection of

5 compounds in MNU-treated mice. Scale bar $=400 \mu \mathrm{m}$. c Magnified images of Rho (red) and

6 PNA (green) expression in (b). The compound group showed increased Rho expression in

7 the outer retinal layer. Scale bar $=100 \mu \mathrm{m}$. d Quantitative results of percentages of Rho-

8 expressing retinal region in the total retinal length (b). Rho-expressing retinal region was

9 significantly longer in the compound group. $n=3$. e Real-time qPCR results of Opsin and

10 Rho expression from the whole retina on day 7 after intravitreal injection of compounds. Rho

11 expression was significantly increased by four compounds. $n=4$. $f$ and $g$ TUNEL staining (f)

12 and quantification of TUNEL-positive photoreceptor cells (g) on day 3 after intravitreal

13 injection of compounds. There is no difference in photoreceptor cell death between the two

14 groups. $\mathrm{n}=3$. Scale bar $=100 \mu \mathrm{m}$. $\mathbf{h}$ Td-Tomato (red) was co-expressed with Rho (green) in

15 the outer retinal layer on day 7 after intravitreal injection of compounds in MNU-treated

16 GFAP-td-Tomato mice. Scale bar $=50 \mu \mathrm{m}$. i Real-time qPCR results of Rho expression and

17 other retinal neuron-specific markers from td-Tomato-positive cell population on day 7 after

18 intravitreal injection. Rho expression was significantly increased by four compounds. $n=3 . \mathbf{j}$ 
1 PSD95 (white) was also expressed around Rho-positive cells in the OPL. Scale bar $=20 \mu \mathrm{m}$.

2 All data are presented as the mean \pm SEM. ${ }^{*} P<0.05,{ }^{* *} P<0.01$, Student's $t$-test.

4 Figure 4. Improvement of retinal function by MC-derived Rho-positive cells.

5 a Scotopic responses of ERGs on day 7 after intravitreal injection of four compounds. ERG

6 waveforms for the $75 \mathrm{mg} / \mathrm{kg} \mathrm{MNU}$ group are shown in red, and those for the $30 \mathrm{mg} / \mathrm{kg}$ MNU

7 group are shown in blue. $\mathbf{b}$ Effect of compounds on the a-wave in the $30 \mathrm{mg} / \mathrm{kg} \mathrm{MNU}$ group.

8 The amplitude of the a-waves was significantly improved by compounds. All data are mean

$9 \quad \pm$ SEM. $\mathrm{n}=4,{ }^{*} P<0.05$, Student's $t$-test.

11 Figure 5. Contribution of Rho-positive cells to the suppression of cone cell death in

12 rd10 mice.

13 a Time course of in vivo experiments using rd10 mice. Intravitreal injection of four compounds

14 was performed every 3 days for 2 weeks from 4 weeks of age. b Real time qPCR results of

15 Opsin and Rho expression from whole retina on day 7 after intravitreal injection of

16 compounds. Rho expression significantly increased by four compounds. $n=4$. c and d

17 Immunohistochemistry of Rho (c) and quantitative results of percentages of Rho-expressing

18 retinal region in the total retinal length (d) on day 14 after intravitreal injection of compounds. 
1 Rho expression was clearly increased in the compound group. Scale bar $=200 \mu \mathrm{m} . \mathrm{n}=4$. e

2 Magnified images of Rho (red) expression and bright field (BF) in (c). Scale bar $=100 \mu \mathrm{m}$. $\mathbf{f}$

3 and $\mathbf{g}$ Whole-mount PNA staining ( $\mathbf{f})$ and quantification of PNA-positive cone cells $(\mathbf{g})$ in the

4 outer retinas on day 7 after intravitreal injection of 4 compounds. $n=3$, Scale bar $=50 \mu \mathrm{m}$.

5 h Real-time qPCR result of NXNL1 on day 7 after intravitreal injection of 4 compounds.

6 NXNL1 expression was significantly increased by four compounds. $n=4$. All data are

$7 \quad$ presented as the mean \pm SEM. ${ }^{*} P<0.05$, Student's $t$-test. 


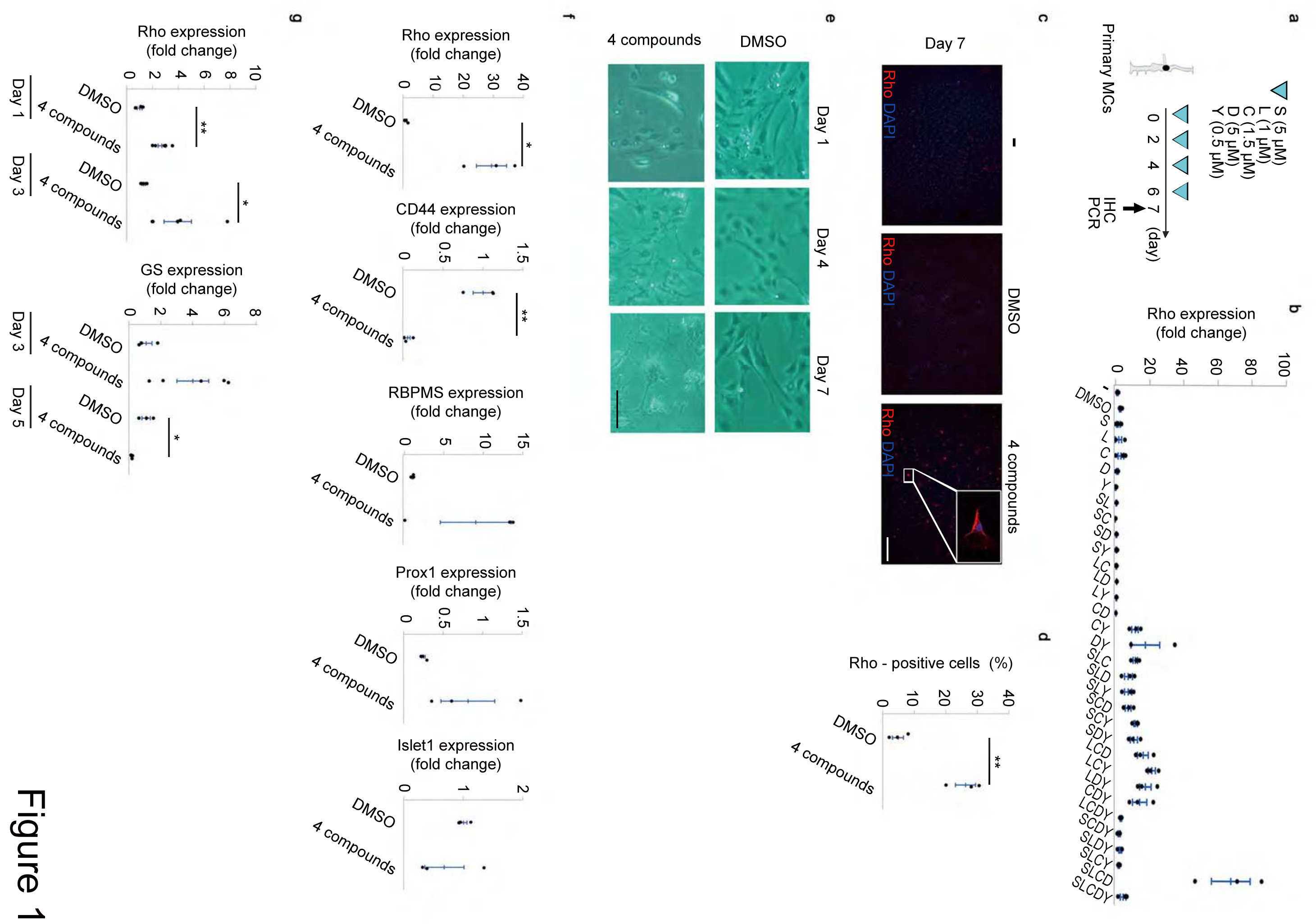




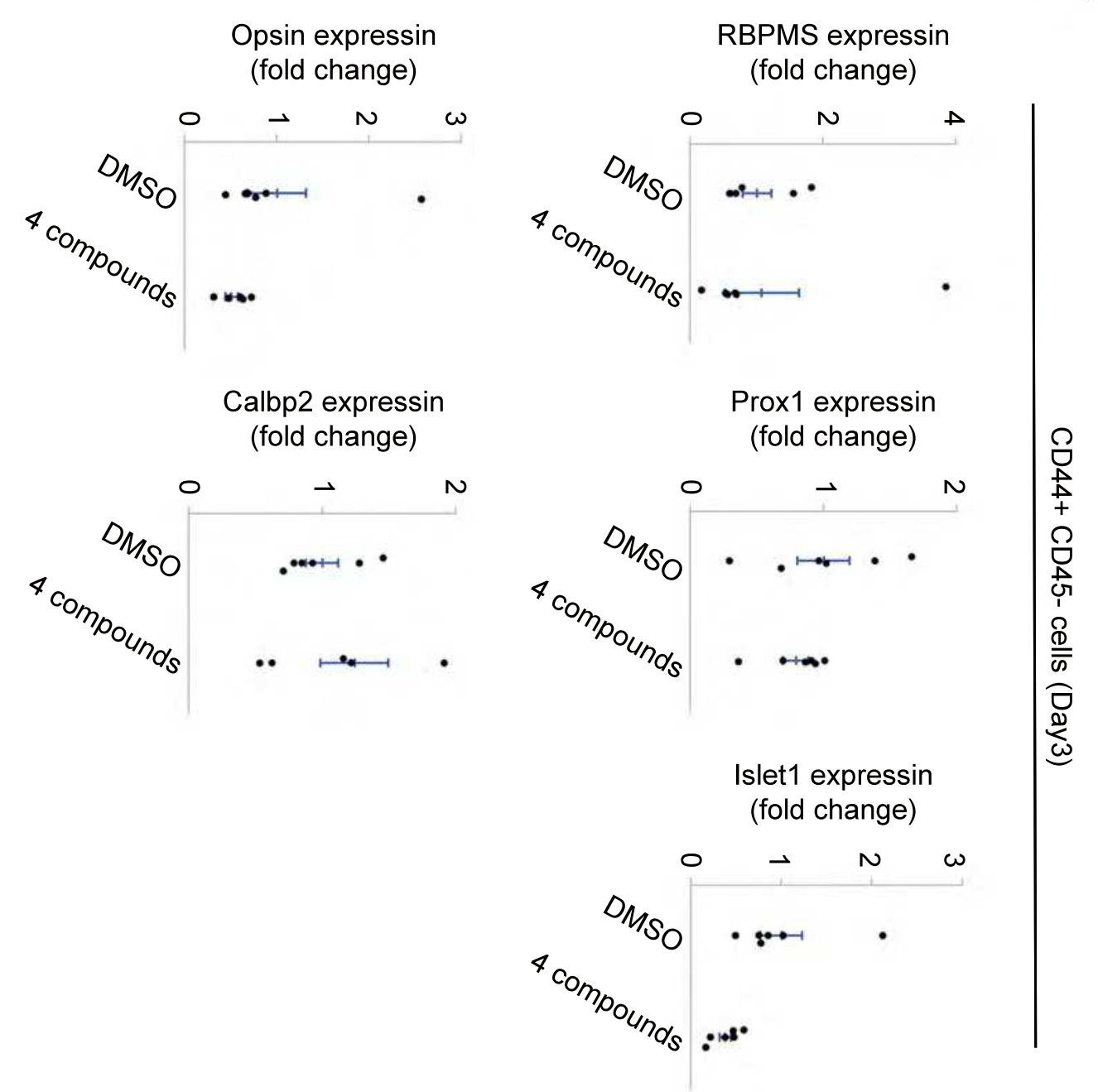

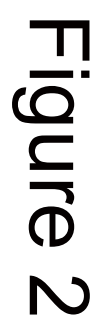
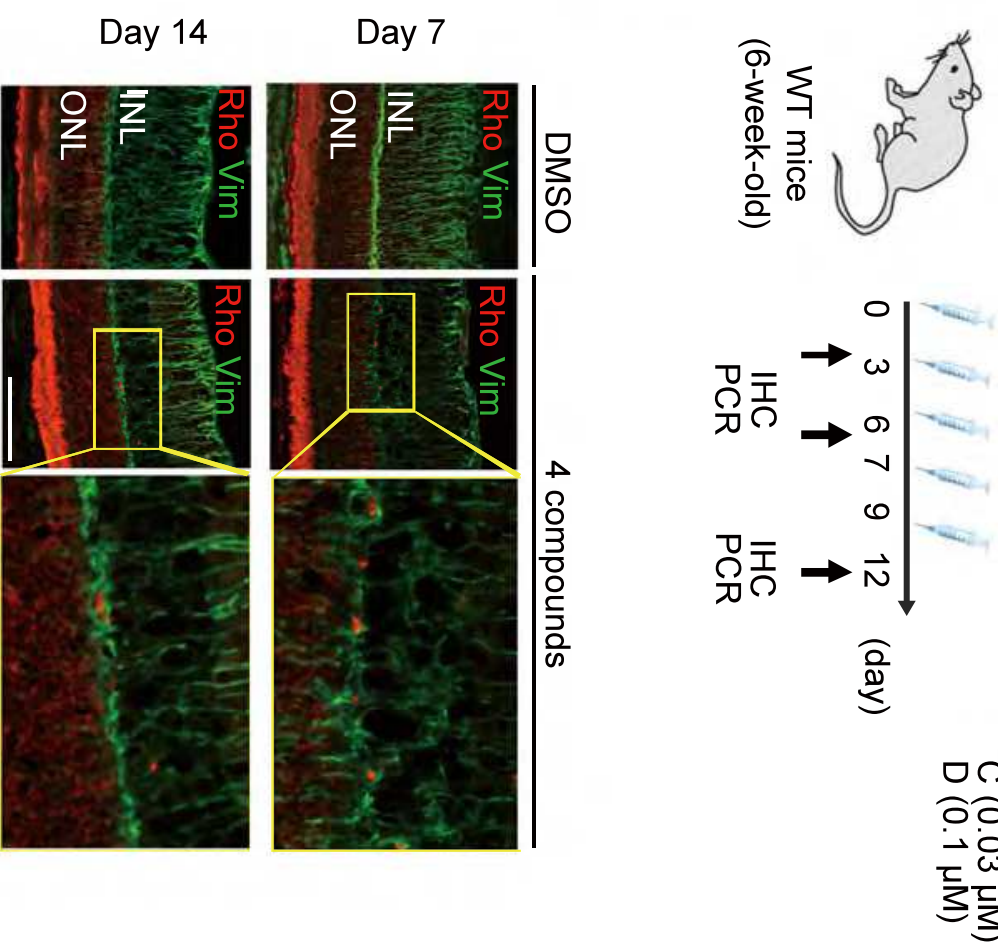

Rho expressin (fold change)

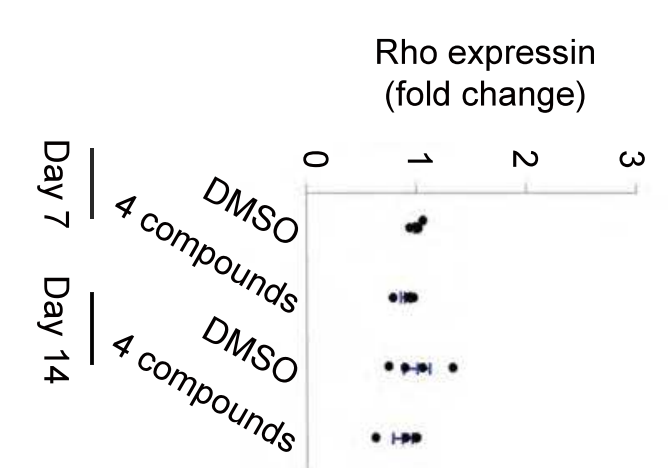

a
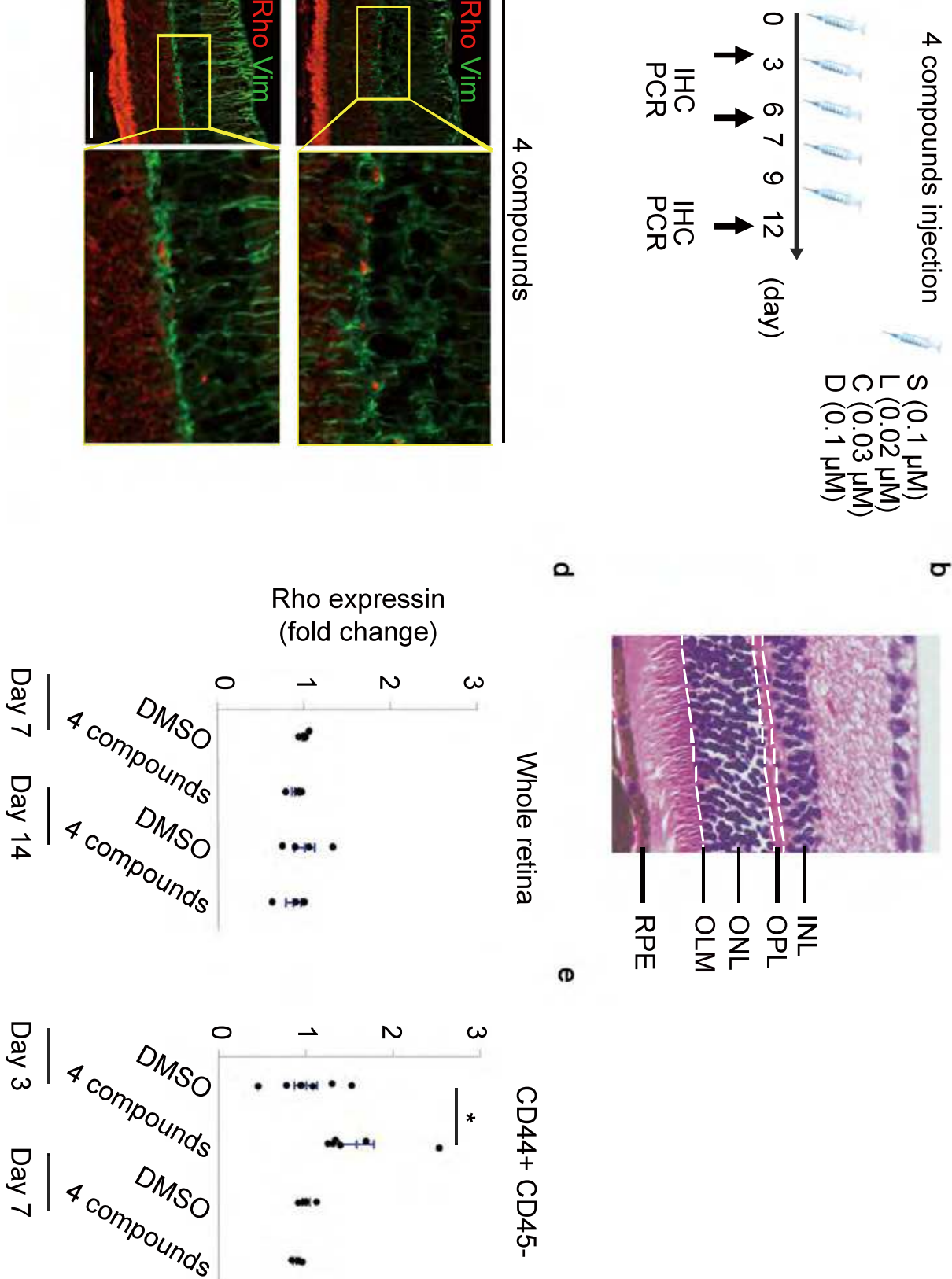
T

Day 7

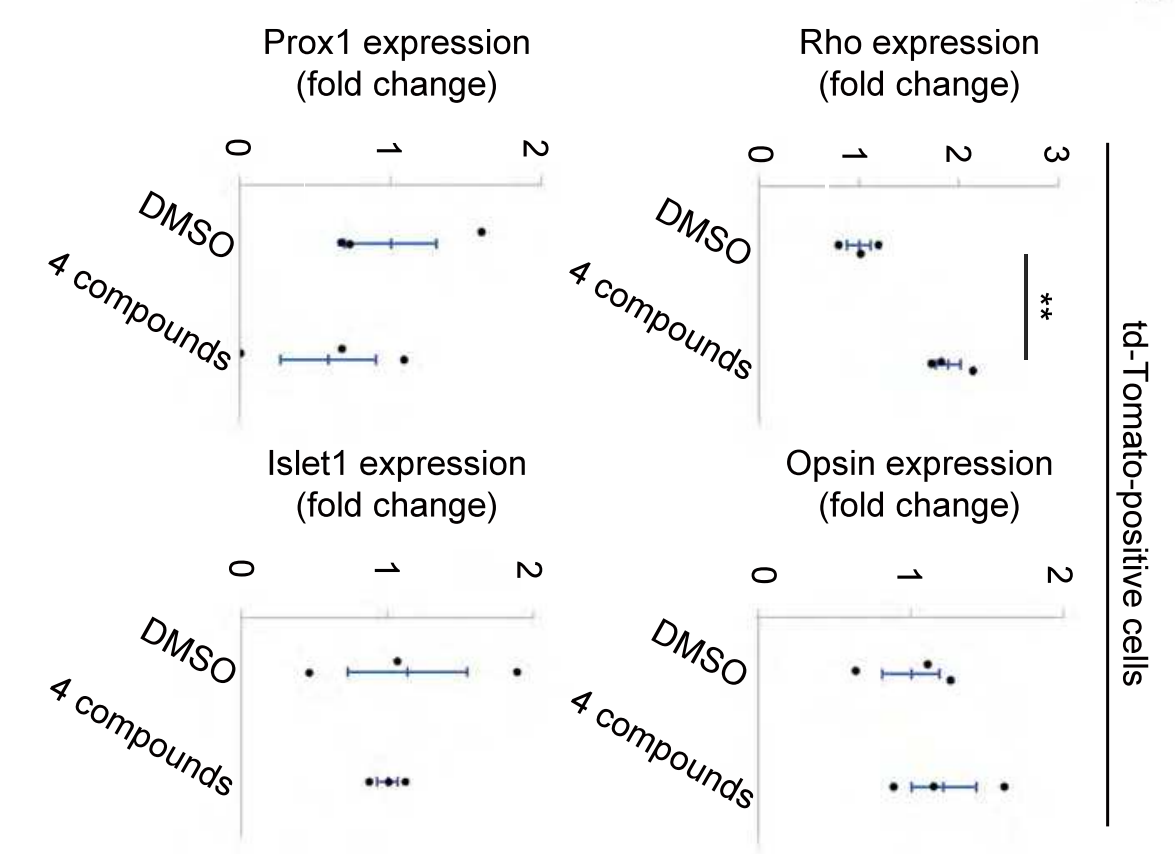

$\begin{array}{cc}\text { Prox1 expression } & \text { Rho expression } \\ \text { (fold change) } & \text { (fold change) }\end{array}$
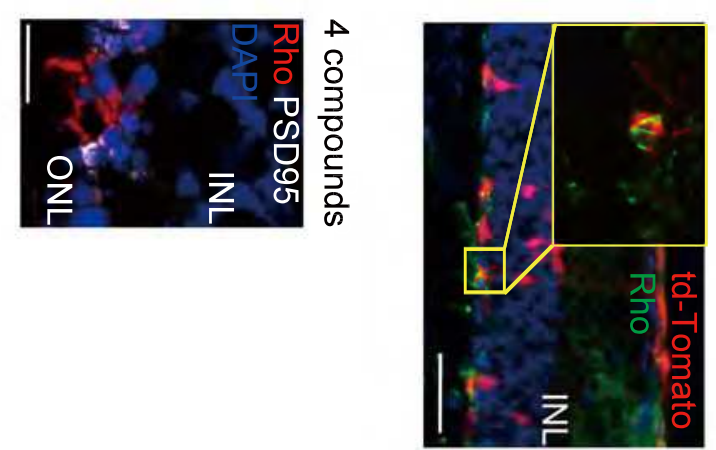

Rho expression

(fold change)
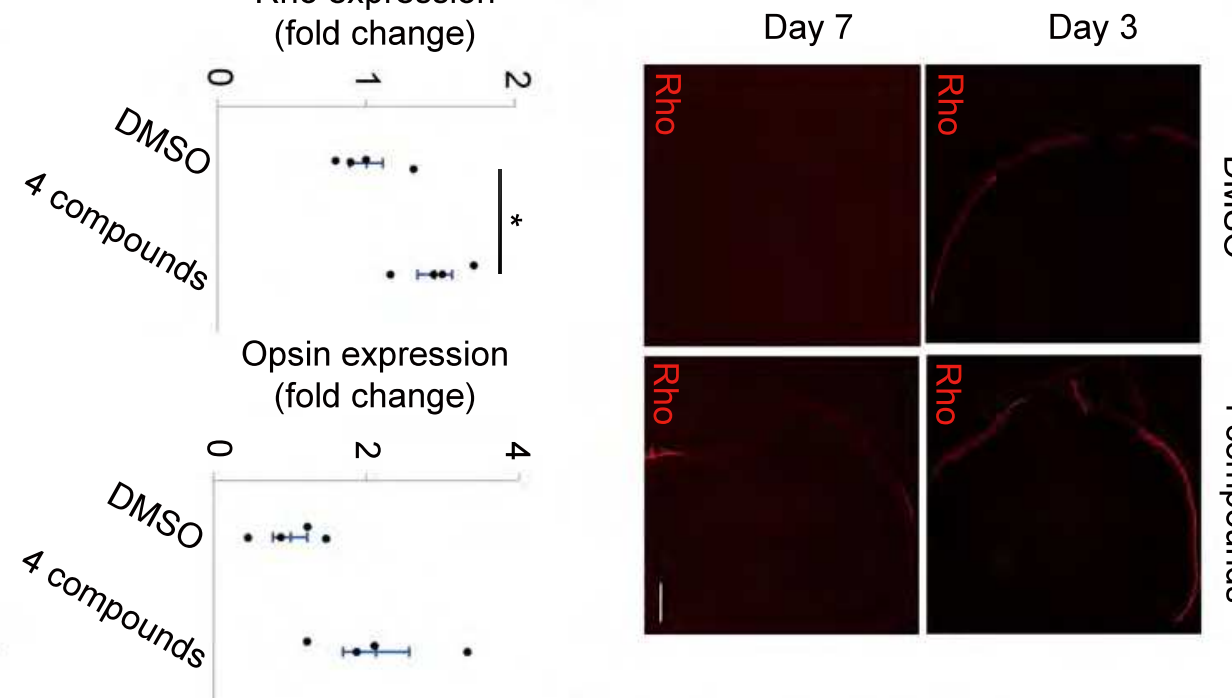

$\therefore$

Opsin expression

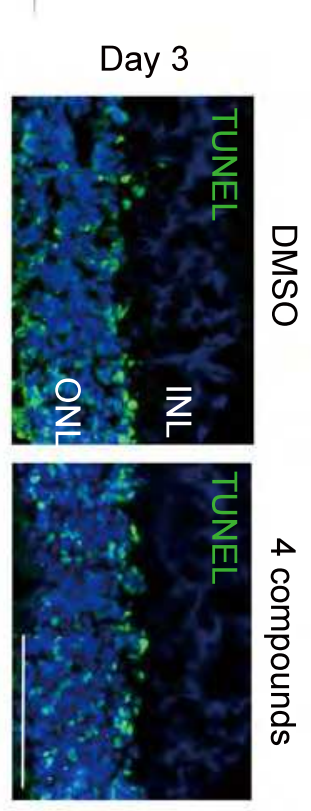

TUNEL-positive cells

/ total cells in $15000 \mu m^{2}$ of ONL (cells)

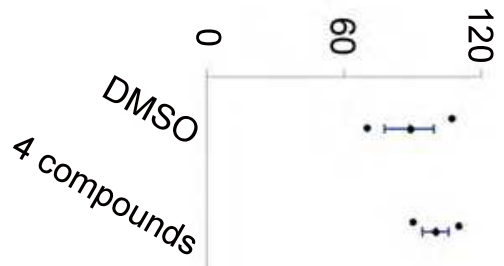

$\frac{\frac{\pi}{\sigma}}{\frac{c}{\bar{C}}}$

Rho-expressing retinal region in the total retinal length (\%)

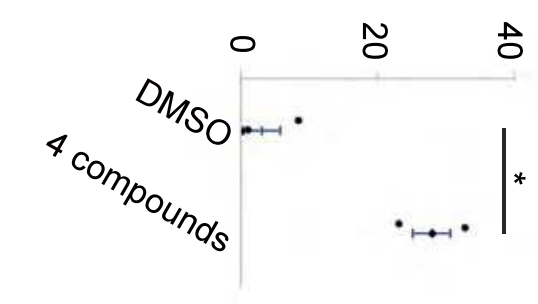

๖

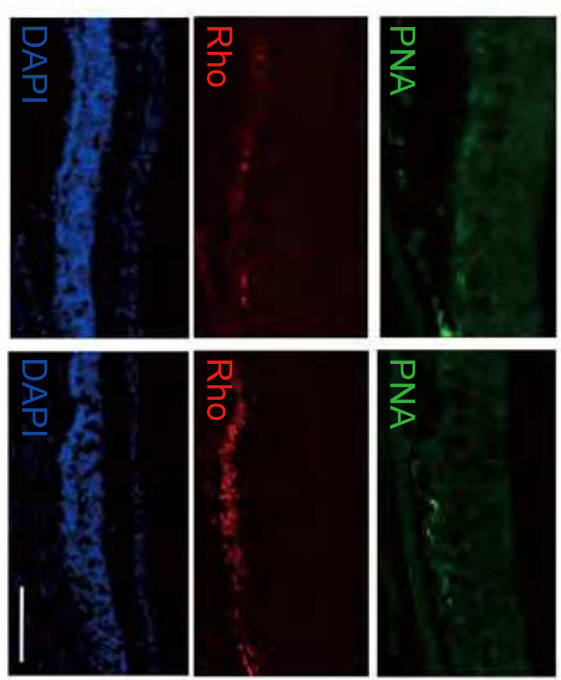

o

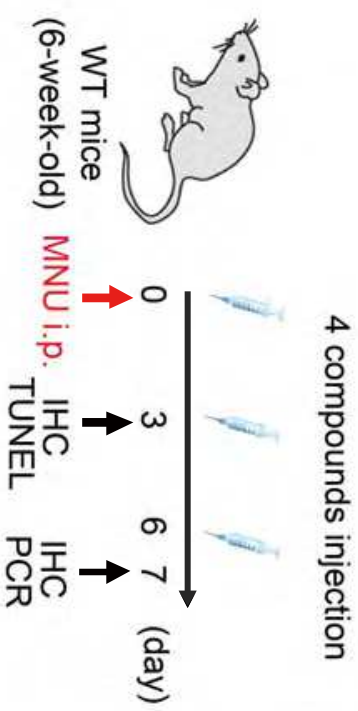

œ

0

व๐Tल 으응 $\rightarrow$ 언그 

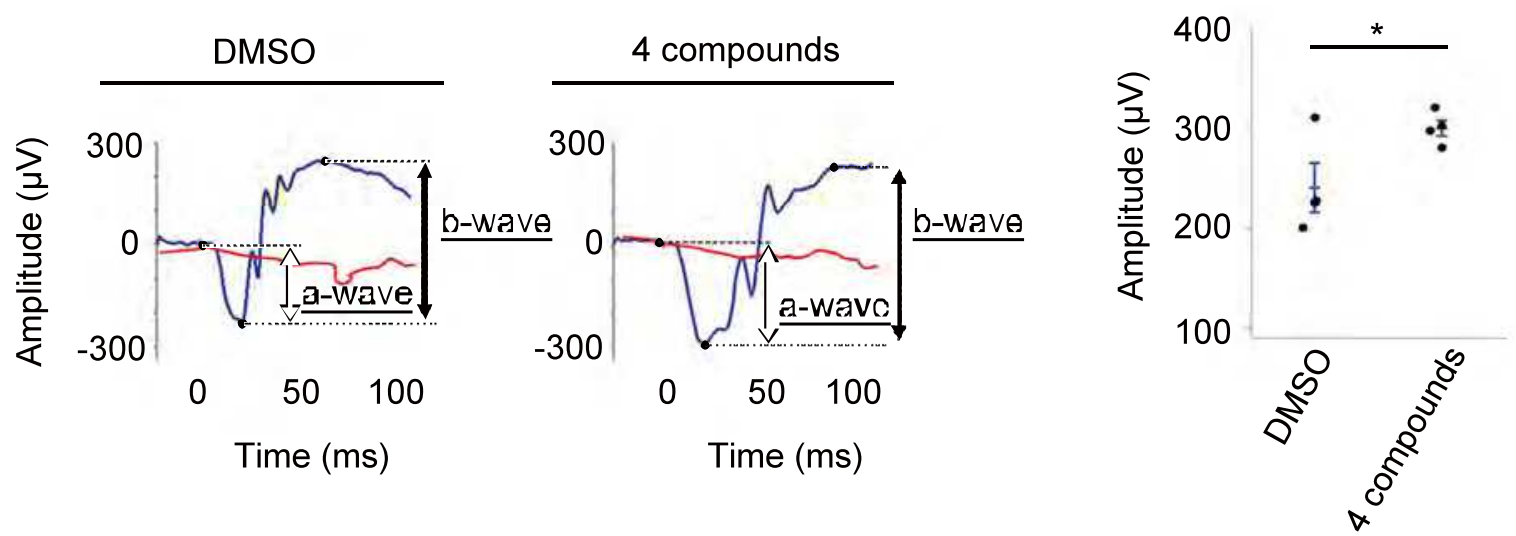
Day 7
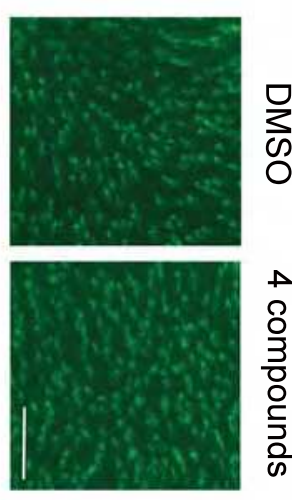

PNA-positive cone cells $1125 \times 125 \mu^{2}$ (cells)

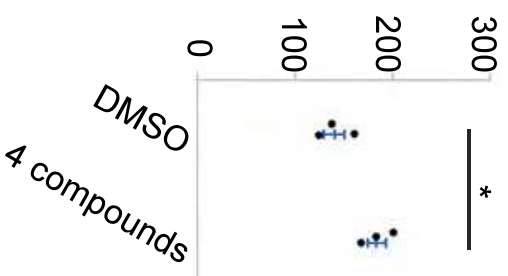

Nxnl1 expresson (fold change)

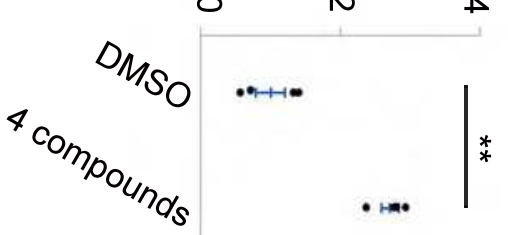

Day 14
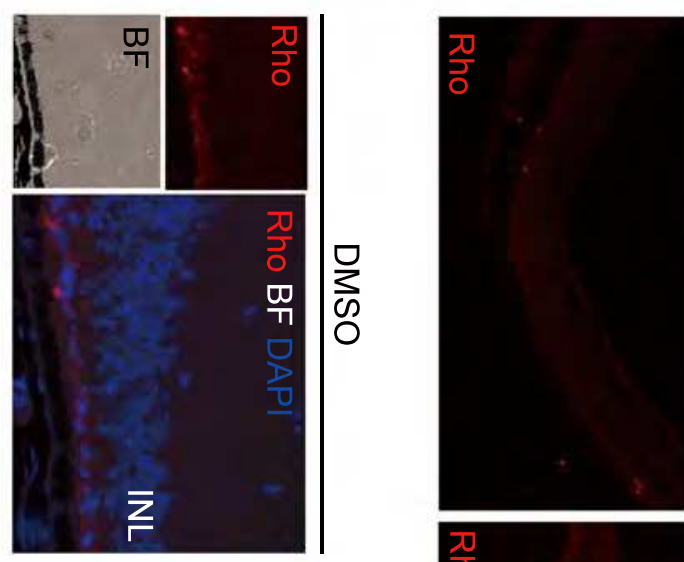

ֻ
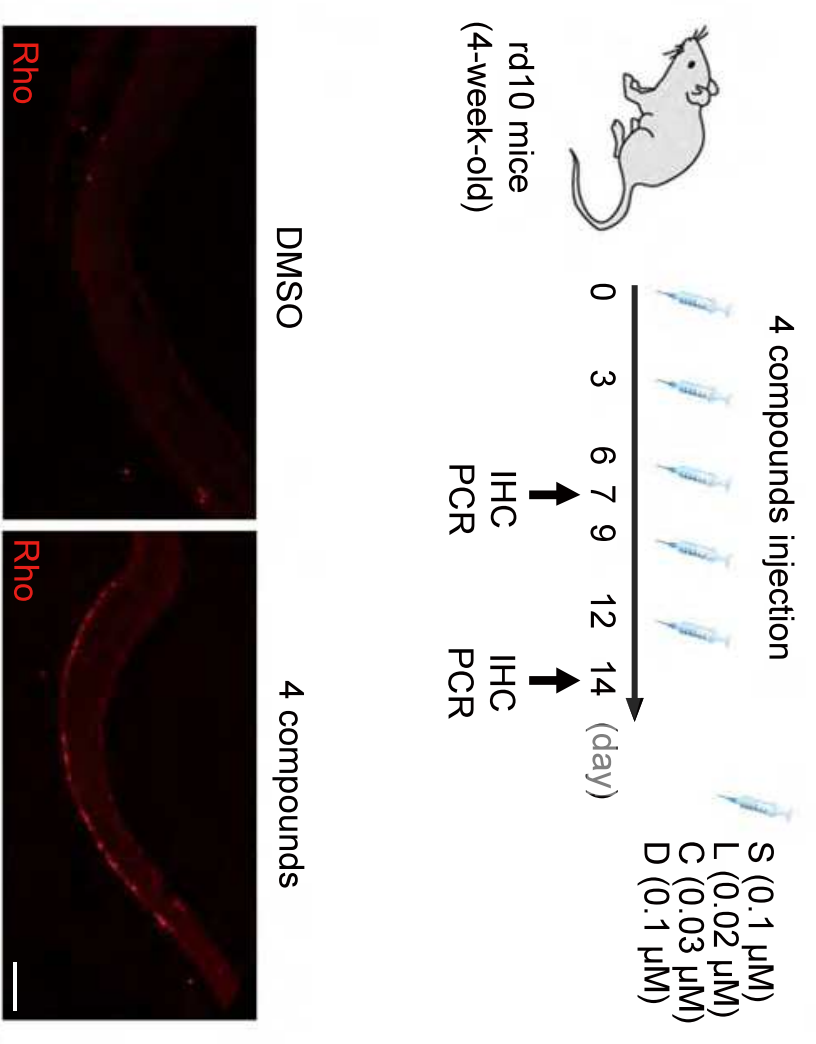

Rho expression (fold change)

Rho-expressing retinal region in the total retinal length (\%)
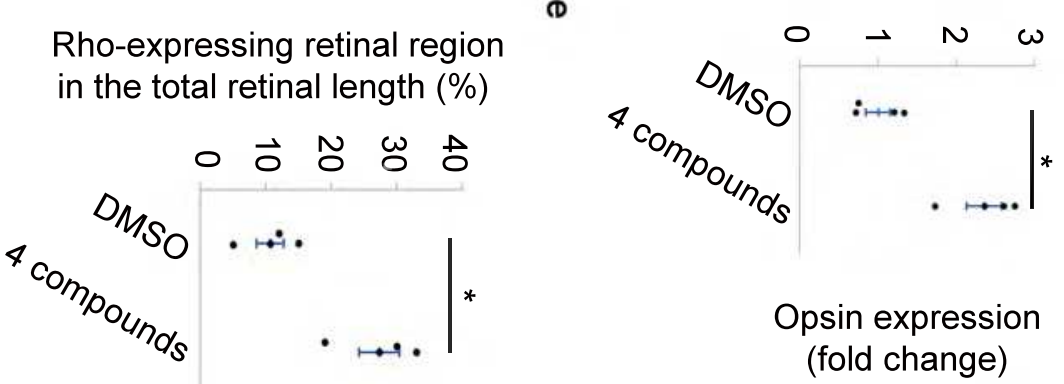

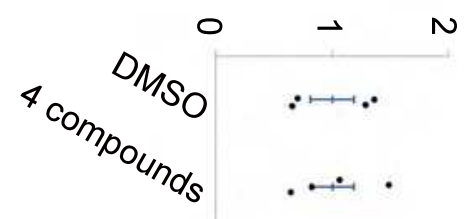




\section{Supplementary Files}

This is a list of supplementary files associated with this preprint. Click to download.

- supplementaryfile.pdf 